\title{
Surjective H-Colouring over Reflexive Digraphs
}

\author{
Benoît Larose \\ LACIM, Université du Québec a Montréal, Canada
}

Barnaby Martin

Department of Computer Science, Durham University, U.K.

Daniël Paulusma

Department of Computer Science, Durham University, U.K.

\begin{abstract}
The SurJective H-Colouring problem is to test if a given graph allows a vertex-surjective homomorphism to a fixed graph H. The complexity of this problem has been well studied for undirected (partially) reflexive graphs. We introduce endo-triviality, the property of a structure that all of its endomorphisms that do not have range of size 1 are automorphisms, as a means to obtain complexity-theoretic classifications of SURJECTIVE H-COLOURING in the case of reflexive digraphs. Chen [2014] proved, in the setting of constraint satisfaction problems, that SURJECTIVE $\mathrm{H}$-COLOURING is NP-complete if $\mathrm{H}$ has the property that all of its polymorphisms are essentially unary. We give the first concrete application of his result by showing that every endo-trivial reflexive digraph $\mathrm{H}$ has this property. We then use the concept of endo-triviality to prove, as our main result, a dichotomy for SuRJECTIVE H-Colouring when $\mathrm{H}$ is a reflexive tournament: if $\mathrm{H}$ is transitive, then SurJective H-Colouring is in NL, otherwise it is NP-complete. By combining this result with some known and new results we obtain a complexity classification for SurJective H-Colouring when $\mathrm{H}$ is a partially reflexive digraph of size at most 3.
\end{abstract}

2012 ACM Subject Classification Mathematics of computing $\rightarrow$ Graph coloring, Theory of computation $\rightarrow$ Problems, reductions and completeness, Theory of computation $\rightarrow$ Graph algorithms analysis

Keywords and phrases Surjective H-Coloring, Computational Complexity, Algorithmic Graph Theory, Universal Algebra, Constraint Satisfaction

Digital Object Identifier 10.4230/LIPIcs.STACS.2018.49

Funding BL was supported by NSERC and FRQNT. DP was supported by The Leverhulme Trust (RPG-2016-258).

Acknowledgements We thank Jan Bok for fruitful discussions as well as three anonymous reviewers for useful comments.

\section{Introduction}

The classical homomorphism problem, also known as H-COLOURING, involves a fixed structure $\mathrm{H}$, with input another structure $\mathrm{G}$, of the same signature, invoking the question as to whether there is a function from the domain of $\mathrm{G}$ to the domain of $\mathrm{H}$ that is a homomorphism from G to H. The H-Colouring problem is an intensively studied problem, which has additionally attracted attention in its guise of the constraint satisfaction problem (CSP), especially since the seminal paper of Feder and Vardi [14]. Their well-known conjecture, recently proved by Bulatov [4] and Zhuk [32], stated that every $\operatorname{CSP}(\mathrm{H})$ has complexity either in $\mathrm{P}$ or NP-complete, omitting any Ladner-like complexities in between.

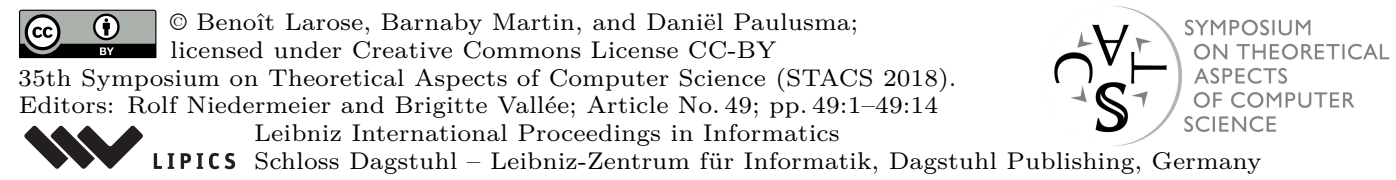


List H-Colouring $\rightarrow$ H-REtraction $\rightarrow$ H-Compaction $\rightarrow$ SurJ H-COlouring $\rightarrow$ H-Colouring

Figure 1 Relations between Surjective H-Colouring and its variants (from [15]). An arrow from one problem to another indicates that the latter problem is polynomial-time solvable for a graph $\mathrm{H}$ whenever the former is polynomial-time solvable for $\mathrm{H}$. Reverse arrows do not hold for the leftmost and rightmost arrows, as witnessed by the reflexive 4-vertex cycle for the rightmost arrow and by any reflexive tree that is not a reflexive interval graph for the leftmost arrow (Feder, Hell and Huang [11] showed that the only reflexive bi-arc graphs are reflexive interval graphs). It is not known whether the reverse direction holds for the two middle arrows.

This paper concerns the computational complexity of the surjective homomorphism problem, also known in the literature as SuRJeCtive H-Colouring $[15,16]$ and H-VERTEXCOMPACTION [30]. This problem requires the homomorphism to be surjective. It is a cousin of the list homomorphism problem and is even more closely related to the retraction and compaction problems. Indeed, the H-COMPACTION problem, hitherto defined only for graphs $\mathrm{H}$, takes as input a graph $\mathrm{G}$ and asks if there exists a function $f$ from $V(\mathrm{G})$ to $V(\mathrm{H})$ so that for each non-loop edge $(x, y) \in E(\mathrm{H})$ (i.e. with $x \neq y$ ), there exists $u, v \in V(\mathrm{G})$ so that $f(u)=x$ and $f(v)=y$. Thus, compaction can be seen as the edge-surjective homomorphism problem. ${ }^{1}$ The problem H-RETRACTION takes as input a superstructure G of H and asks whether there is a homomorphism from $\mathrm{G}$ to $\mathrm{H}$ that is the identity on $\mathrm{H}$. The H-RETRACTION problem is polynomially equivalent with a special type of $\operatorname{CSP}, \operatorname{CSP}\left(\mathrm{H}^{\prime}\right)$, where $\mathrm{H}^{\prime}$ is $\mathrm{H}$ decorated with constants naming the elements of its domain. Feder and Vardi [14] showed that the task of classifying the complexities of the retraction problems is equivalent to that for the CSPs. Hence, owing to [4, 32], H-RETRACTION has now been fully classified.

The list homomorphism problem, List H-COLOURING, allows one to express restricted lists for each of the input structure's elements, that are the only domain elements permitted in a solution homomorphism. LIST H-COLOURING is also a special type of CSP, CSP $\left(\mathrm{H}^{\prime}\right)$, where $\mathrm{H}^{\prime}$ is $\mathrm{H}$ replete with all possible unary relations over the domain of $\mathrm{H}$. Historically, the complexities of List H-COLOURING were the first to be settled by Bulatov [3], following important earlier work on graphs $[9,10,11]$.

In contrast to the situation for H-Colouring, List H-ColOuRING and H-RETRACTION, the complexity classifications for H-COMPACTION and SURJECTIVE H-COLOURING are far from settled, and there are concrete open cases (see 3-No-RAINBOw-ColOURING in the survey [2]). Obtaining NP-hardness for compaction and surjective homomorphism problems appears to be especially challenging. The complexity-theoretic relationship between these various problems is drawn in Figure 1. At present it is not known whether there is a graph $\mathrm{H}$ so that H-Retraction, H-Compaction and Surjective H-Colouring do not have the same complexity up to polynomial time reduction (see $[15,29]$ ).

Nevertheless classification results for SuRJEctive H-Colouring have tried to keep pace with similar ones for H-RETRACTION. In [12] it is proved, among partially reflexive pseudoforests $\mathrm{H}$, where the problem H-REtraction splits between P and NP-complete. A similar classification for SuRJECTIVE H-Colouring over partially reflexive forests can be inferred from the classification for partially reflexive trees in [16]. The quest for a classification for H-Compaction and Surjective H-Colouring over pseudoforests is ongoing, but for both problems already the reflexive 4-cycle took some time to classify [24, 27], as well as the irreflexive 6-cycle [28, 31].

1 Except for the treatment of self-loops, which appears to be an idiosyncrasy that plays no vital role in computational complexity. For some history of the definition see [27]. 
The above results are for undirected graphs, whereas we focus on digraphs. A known classification for H-RETRACTION comes for irreflexive semicomplete digraphs H. In [1] BangJensen, Hell, and MacGillivray proved that H-Colouring is always in P or is NP-complete if $\mathrm{H}$ is irreflexive semicomplete. This is a fortiori a classification for H-RETRACTION since semicomplete digraphs are cores (all endomorphisms are automorphisms), which ensures that H-Colouring and H-Retraction are polynomially equivalent. For irreflexive semicomplete digraphs $\mathrm{H}$, the classification for SuRJECTIVE H-Colouring can be read trivially from that for H-Colouring, and they are the same. An obvious next place to look is at the situation if $\mathrm{H}$ is reflexive semicomplete, where surely the classifications will not be the same as H-ColOuring is trivial in this case.

Reflexive tournaments form an important subclass of the class of reflexive semicomplete graphs and are well-understood algebraically [19]. In particular, the classification for H-RETRACTION where $\mathrm{H}$ is a reflexive tournament can be inferred from the algebraic characterisation from [19]: for a reflexive tournament $\mathrm{H}$, the H-RETRACTION problem is in $\mathrm{NL}$ if $\mathrm{H}$ is transitive, and it is NP-complete otherwise. This raises the question whether the same holds for SurJeCtive H-Colouring and whether we can develop algebraic methods further to prove this. In fact, the algebraic method is by now well known for CSPs and their relatives, including its use with digraphs; see the recent survey [20]. However, the algebraic method is not so far advanced for surjective homomorphism problems. So far it only exists in the work of Chen [7], who proved that SuRJECTIVE H-Colouring is NP-complete if H has the property that all of its polymorphisms depend only on one variable, that is, are essentially unary. Chen's result has not yet been put to work (even on toy open problems) and a key driver for our research has been to find, in the wild, a place for its application.

Our Results. We give, for the first time, complexity classifications for SuRJECTIVE HColouring for digraphs instead of undirected graphs. To prove our results, we further develop algebraic machinery to tackle surjective homomorphism problems. That is, in Section 2 we introduce, after giving the necessary terminology, the concept of endo-triviality. We show how this concept is closely related to some known algebraic concepts and explore its algorithmic consequences in the remainder of our paper.

Firstly, in Section 3, we prove that a reflexive digraph $\mathrm{H}$ that is endo-trivial has the property that all of its polymorphisms are essentially unary. Combining this result with the aforementioned result of Chen [7] immediately yields that SuRJECTIVE H-Colouring is NP-complete for any such digraph $\mathrm{H}$. This is the first concrete application of Chen's result to settle a problem of open complexity; it shows, for instance, that SuRJECTIVE H-CoLOURING is NP-complete if $\mathrm{H}$ is a reflexive directed cycle on $k \geq 3$ vertices. As the case $k \leq 2$ is trivial, this gives a classification of SURJECTIVE H-COLOURING for reflexive directed cycles, which we believe form a natural class of digraphs to consider given the results in $[24,31]$.

Secondly, in Section 4 we give a complexity classification for SurJective H-Colouring, when $\mathrm{H}$ is a reflexive tournament. We use endo-triviality in an elaborate and recursive encoding of an NP-hard retraction problem within SuRJECTIVE H-Colouring. In doing this, we show that on this class, the complexities of SuRJECTIVE H-COLOURING and HRETRACTION coincide.

Finally, our results enable us to give a complexity classification for SuRJEctive HColouring when $\mathrm{H}$ is a partially reflexive digraph of size at most 3 . In doing this, we show that on this class, the complexities of SURJECTIVE H-COLOURING and H-RETRACTION coincide. We are not aware of an existing classification for H-RETRACTION on this class, but we do build on one existing for List H-ColOURING from [13]. 


\section{Preliminaries}

Let $[n]:=\{1, \ldots, n\}$. For a $k$-tuple $\bar{t}$ and $i \in[k]$, let $\bar{t}[i]$ be the $i$ th entry in $\bar{t}$. In a digraph $\mathrm{G}$, a forward- (resp., backward-) neighbour (or adjacent) to a vertex $u \in V(\mathrm{G})$ is another vertex $v \in V(\mathrm{G})$ so that $(u, v) \in E(\mathrm{G})$ (resp., $(v, u) \in E(\mathrm{G})$ ). The out-degree and in-degree of a vertex are the number of its forward-neighbours and backward-neighbours, respectively. A vertex with out-degree and in-degree both 0 is said to be isolated. A vertex with a self-loop is reflexive and otherwise it is irreflexive. A digraph is (ir)reflexive if all its vertices are (ir)reflexive.

The directed path on $k$ vertices is the digraph with vertices $u_{0}, \ldots, u_{k-1}$ and edges $\left(u_{i}, u_{i+1}\right)$ for $i=0, \ldots, k-2$. The directed cycle on $k$ vertices is obtained from the directed path on $k$ vertices after adding the edge $\left(u_{k-1}, u_{0}\right)$. A digraph $\mathrm{G}$ is strongly connected if for all $u, v \in V(\mathrm{G})$ there is a directed path in $E(\mathrm{G})$ from $u$ to $v$ (note that we take this to include the situation $u=v$, but for reflexive graphs the distinction is moot). A digraph is weakly connected if its symmetric closure (underlying undirected graph) is connected. A double-edge in a digraph $\mathrm{G}$ consists in a pair of distinct vertices $u, v \in V(\mathrm{G})$, so that $(u, v),(v, u) \in E(\mathrm{G})$. A digraph $\mathrm{G}$ is semicomplete if for every two distinct vertices $u$ and $v$, at least one of $(u, v)$, $(v, u)$ belongs to $E(\mathrm{G})$. A digraph $\mathrm{G}$ is a tournament if for every two distinct vertices $u$ and $v$, exactly one of $(u, v),(v, u)$ belongs to $E(\mathrm{G})$. We demand our tournaments have more than one vertex (to rule out certain trivial cases in proofs). A reflexive tournament $\mathrm{G}$ is transitive if for every triple of vertices $u, v, w$ with $(u, v),(v, w) \in E(\mathrm{G})$, also $(u, w)$ belongs to $E(\mathrm{G})$. A digraph $\mathrm{F}$ is a subgraph of a digraph $\mathrm{G}$ if $V(\mathrm{~F}) \subseteq V(\mathrm{G})$ and $E(\mathrm{~F}) \subseteq E(\mathrm{G})$. It is induced if $E(\mathrm{~F})$ coincides with $E(\mathrm{G})$ restricted to pairs containing only vertices of $V(\mathrm{~F})$. A subtournament is an induced subgraph of a tournament (note that this is a fortiori a tournament). All subgraphs we consider in this paper will be induced.

A homomorphism from a digraph $\mathrm{G}$ to a digraph $\mathrm{H}$ is a function $f: V(\mathrm{G}) \rightarrow V(\mathrm{H})$ so that for all $u, v \in V(\mathrm{G})$ with $(u, v) \in E(\mathrm{G})$ we have $(f(u), f(v)) \in E(\mathrm{H})$. We say that $f$ is (vertex)-surjective if for every vertex $x \in V(\mathrm{H})$ there exists a vertex $u \in V(\mathrm{G})$ with $f(u)=x$. Let $\mathrm{H}$ be a digraph. A homomorphic image of $\mathrm{H}$ is a digraph $\mathrm{H}^{\prime}$ so that there is a surjective homomorphism $h: \mathrm{H} \rightarrow \mathrm{H}^{\prime}$ in which, for all $\left(x^{\prime}, y^{\prime}\right) \in E\left(\mathrm{H}^{\prime}\right)$ there exists $(x, y) \in E(\mathrm{H})$ so that $x^{\prime}=h(x)$ and $y^{\prime}=h(y)$. That is, $h$ is vertex- and edge-surjective.

The direct product of two digraphs $\mathrm{G}$ and $\mathrm{H}$, denoted $\mathrm{G} \times \mathrm{H}$, has vertex set $V(\mathrm{G}) \times V(\mathrm{H})$ and edges $\left((x, y),\left(x^{\prime}, y^{\prime}\right)\right)$ exactly when $\left(x, x^{\prime}\right) \in E(\mathrm{G})$ and $\left(y, y^{\prime}\right) \in E(\mathrm{H})$. This product is associative and commutative, up to isomorphism, and spawns a natural power. A $k$-ary polymorphism of $\mathrm{G}$ is a function $f: \mathrm{G}^{k} \rightarrow \mathrm{G}$ so that when $\left(x_{1}, y_{1}\right), \ldots,\left(x_{k}, y_{k}\right) \in E(\mathrm{G})$ then $\left(f\left(x_{1}, \ldots, x_{k}\right), f\left(y_{1}, \ldots, y_{k}\right)\right) \in E(\mathrm{G})$. A polymorphism of $\mathrm{G}$ can be seen as a homomorphism from the $k$ th (direct) power of $\mathrm{G}, \mathrm{G}^{k}$, to $\mathrm{G}$. A polymorphism $f$ is idempotent if for all $x \in V(\mathrm{G}), f(x, \ldots, x)=x$. The $k$-ary $i$ th projection, for $i \in[k]$, is the polymorphism $\pi_{k}^{i}$ given by $\pi_{k}^{i}\left(x_{1}, \ldots, x_{k}\right)=x_{i}$. A $k$-ary operation $f$ is called essentially unary if there exists a unary operation $g$ and $i \in[k]$ so that $f\left(x_{1}, \ldots, x_{k}\right)=g\left(x_{i}\right)$ for all $\left(x_{1}, \ldots, x_{k}\right) \in \mathrm{G}^{k}$.

Let $\mathrm{G}$ be a digraph. An endomorphism of $\mathrm{G}$ is a homomorphism from $\mathrm{G}$ to itself. An endomorphism $e$ of $\mathrm{G}$ is a constant map if there exists a vertex $v \in V(\mathrm{G})$ such that $e(u)=v$ for all $u \in V(\mathrm{G})$. The endomorphism digraph $\mathrm{G}^{\mathrm{G}}$ has as its vertices the endomorphisms of $\mathrm{G}$, and there is an edge $(f, g) \in E\left(\mathrm{G}^{\mathrm{G}}\right)$ between endomorphisms $f$ and $g$ if and only if for every edge $(x, y) \in E(\mathrm{G})$, we have that $(f(x), g(y)) \in E(\mathrm{G})$. We note that $\mathrm{G}^{\mathrm{G}}$ is reflexive when $\mathrm{G}$ is reflexive and also make two more observations. The first one follows directly from the definition of $\mathrm{G}^{\mathrm{G}}$ as well. The second one can, for example, be found in Section 5.2 of [21].

- Lemma 1. If $\left(f_{1}, g_{1}\right) \in E\left(\mathrm{G}^{\mathrm{G}}\right)$ and $\left(f_{2}, g_{2}\right) \in E\left(\mathrm{G}^{\mathrm{G}}\right)$, then $\left(\left(f_{1} \circ f_{2}\right),\left(g_{1} \circ g_{2}\right)\right) \in E\left(\mathrm{G}^{\mathrm{G}}\right)$. 


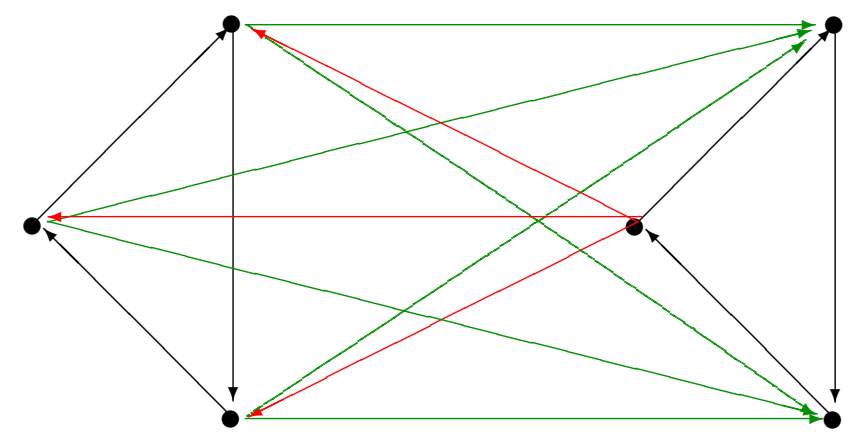

Figure 2 A tournament on six vertices (self-loops are not drawn), which retracts to the directed 3-cycle (in black) on the right-hand side, but not to the one on the left-hand side (in black as well). However, there is no endomorphism that maps the left-hand one isomorphically to the right. We can use this tournament to build a structure that is a counterexample to the generalisation of Lemma 5 stating that endo-trivial and retract-trivial coincide. Let us label the vertices in the tournament: $\alpha, \beta, \gamma$ (left-hand $\mathrm{DC}_{3}^{*}$, clockwise from bottom) and 0,1,2 (right-hand $\mathrm{DC}_{3}^{*}$, clockwise from bottom). Let us build a structure B by augmenting a new 6 -ary relation with tuples in $\{(\alpha, \beta, \gamma, 0,1,2),(\alpha, \alpha, \alpha, \alpha, \beta, \gamma),(\alpha, \alpha, \alpha, \alpha, \alpha, \alpha)\}$. The structure B is retract-trivial but is not endo-trivial, since it has an interesting endomorphism that takes $(\alpha, \beta, \gamma, 0,1,2)$ to $(\alpha, \alpha, \alpha, \alpha, \beta, \gamma)$.

- Lemma 2. Let $\mathrm{G}$ and $\mathrm{H}$ be two digraphs. Let $\phi$ be a homomorphism from $\mathrm{H} \times \mathrm{G}$ to $\mathrm{G}$. Then the function $\psi$ defined by $\psi(x)(u)=\phi(x, u)$ for all $x \in V(\mathrm{H}), u \in V(\mathrm{G})$ is a homomorphism from $\mathrm{H}$ to $\mathrm{G}^{\mathrm{G}}$.

A bijective endomorphism whose inverse is a homomorphism is an automorphism. An endomorphism is non-trivial if it is neither an automorphism nor a constant map. A digraph, all of whose endomorphisms are automorphisms, is termed a core. An endomorphism $e$ of a digraph $\mathrm{H}$ fixes a subset $S \subseteq V(\mathrm{H})$ if $e(S)=S$, that is, $e(x) \in S$ for all $x \in S$, and it fixes a subgraph $\mathrm{F}$ of $\mathrm{H}$ if $e(\mathrm{~F})=\mathrm{F}$. It fixes an induced subgraph $\mathrm{F}$ up to automorphism if $e(\mathrm{~F})$ is an automorphic copy of $\mathrm{F}$ (this is a stronger condition than $e(\mathrm{~F})$ being isomorphic to $\mathrm{F}$ ). An endomorphism $r$ of $\mathrm{G}$ is a retraction of $\mathrm{G}$ if $r$ is the identity on the image $r(\mathrm{G})$ (thus a retraction must have at least one fixed point).

Endo-triviality and Retract-triviality. We now define the key concept of endo-triviality and the closely related concept of retract-triviality.

- Definition 3. A digraph is endo-trivial if all of its endomorphisms are automorphisms or constant maps.

The concept of endo-triviality also arises from the perspective of the algebra of polymorphisms. An algebra is called minimal if its unary polynomials are either constants or the permutations (see Definition 2.14 in [17]). For reflexive digraphs, polynomials and polymorphisms coincide. In other words, a reflexive digraph is endo-trivial if and only if its associated algebra of polymorphisms is minimal.

We will also need the following closely related concept.

- Definition 4. A digraph is retract-trivial if all of its retractions are the identity or constant maps.

The concept of retract-triviality also appears in the algebraic theory but has, as far as we are aware, not been studied in a combinatorial setting. An algebra is term-minimal if the only retractions in its clone of terms are the identity and constants (see [26]). A reflexive digraph 
is retract-minimal if its associated algebra of polymorphisms is term-minimal. It follows that on reflexive digraphs, the concepts of retract-minimality and retract-triviality coincide.

We note that every endo-trivial structure is also retract-trivial. However, the reverse implication is not necessarily true: in Figure 2 we give an example of a structure that is retract-trivial but not endo-trivial. This example is based on a digraph but is not itself a digraph. It is also possible to construct a retract-trivial digraph that is not endo-trivial [25], but on reflexive tournaments both concepts do coincide.

- Lemma 5. A reflexive tournament is endo-trivial if and only if it is retract-trivial.

Proof. (Forwards.) Trivial. (Backwards.) By contraposition, suppose $e$ is a non-trivial endomorphism of a reflexive tournament $\mathrm{H}$. Consider $e(\mathrm{H})$ and build some function $e^{-1}$ from $e(\mathrm{H})$ to $\mathrm{H}$ by choosing $e^{-1}(y)=x$ if $e(x)=y$ arbitrarily. Since $\mathrm{H}$ is a (reflexive) tournament, $e^{-1}$ is an isomorphism, whereupon $e^{-1} \circ e$ is the identity automorphism when restricted to some subtournament $\mathrm{H}_{0}$ of $\mathrm{H}$. Hence $e^{-1} \circ e$ is a non-trivial retraction of $\mathrm{H}$ (to $\mathrm{H}_{0}$ ).

\section{Essential Unarity and a Dichotomy for Reflexive Directed Cycles}

In this section we give the first concrete application, of which we are aware, of the aforementioned result of Chen, formally stated below.

- Theorem 6 (Corollary 3.5 in [7]). Let $\mathrm{H}$ be a finite structure whose universe $V(\mathrm{H})$ has size strictly greater than 1 . If each polymorphism of $\mathrm{H}$ is essentially unary, then SURJECTIVE $\mathrm{H}$-COLOURING is NP-complete.

In order to this, we make use of the endomorphism graph and a result from Mároti and Zádori [23]. Let $i d_{\mathrm{H}}$ denote the identity map on a digraph $\mathrm{H}$.

- Lemma 7 (Lemma 2.2 in [23]). Let $\mathrm{H}$ be a reflexive digraph. If $\left(i d_{\mathrm{H}}, f\right) \in E\left(\mathrm{H}^{\mathrm{H}}\right)$, where $f$ is different from $i d_{\mathrm{H}}$, then $\mathrm{H}$ has a non-surjective retraction $r$ such that $\left(i d_{\mathrm{H}}, r\right) \in E\left(\mathrm{H}^{\mathrm{H}}\right)$.

The following lemma is crucial and will be of use in the next section as well.

- Lemma 8. Let $\mathrm{H}$ be a retract-trivial reflexive digraph with at least three vertices. Then

1. $\mathrm{H}$ has no double edge;

2. $\mathrm{H}$ is strongly connected; and

3. the automorphisms of $\mathrm{H}$ are isolated vertices in $\mathrm{H}^{\mathrm{H}}$.

We use Lemma 8 to obtain the following structural result.

- Theorem 9. Let $\mathrm{H}$ be an endo-trivial reflexive digraph with at least three vertices. Then every polymorphism of $\mathrm{H}$ is essentially unary.

Proof. Since $\mathrm{H}$ is endo-trivial, $\mathrm{H}$ is retract-trivial. Hence, by Lemma $8, \mathrm{H}$ is strongly connected, and furthermore the automorphisms of $\mathrm{H}$ are isolated vertices of $\mathrm{H}^{\mathrm{H}}$. As $\mathrm{H}$ is endo-trivial, this means that $\mathrm{H}^{\mathrm{H}}$ is the disjoint union of a copy of $\mathrm{H}$ that corresponds to the constant maps and a set of isolated vertices, one for each automorphism of $\mathrm{H}$. Suppose for a contradiction that there exists an an $n$-ary polymorphism $f$ of $\mathrm{H}$ which is not essentially unary. We may without loss of generality assume that $f$ depends on all of its $n$ variables, where $n \geq 2$. By Lemma 2, the mapping $F: \mathrm{H}^{n-1} \rightarrow \mathrm{H}^{\mathrm{H}}$ defined by $F\left(x_{1}, \ldots, x_{n-1}\right)(y)=f\left(x_{1}, \ldots, x_{n-1}, y\right)$ is a homomorphism. Since $\mathrm{H}$ is strongly connected, so is $\mathrm{H}^{n-1}$, and hence so is the image of $F$ in $\mathrm{H}^{\mathrm{H}}$. Thus this image is either contained in the component of constants, in which case $f$ does not depend on its last variable, else it is a singleton, in which case $f$ does not depend on any of its first $n-1$ variables. 
Combining Theorems 6 and 9 yields the main result of this section.

- Corollary 10. If $\mathrm{H}$ is an endo-trivial reflexive digraph on at least three vertices, then SurJective H-Colouring is NP-complete.

Let $\mathrm{DC}_{k}^{*}$ denote the reflexive directed cycle on $k$ vertices, which is readily seen to be endo-trivial. Corollary 10 yields the following dichotomy for reflexive directed cycles after noting that SurJective $\mathrm{DC}_{k}^{*}$-COLOURING is trivial for $k \leq 2$.

- Corollary 11. SurjeCtive $\mathrm{DC}_{k}^{*}$-Colouring is in $\mathrm{L}$ if $k \leq 2$ and NP-complete if $k \geq 3$.

It is not difficult to construct endo-trivial reflexive tournaments other than reflexive directed cycles. In the next section though we give a combinatorial NP-hardness proof for SurJective H-Colouring whenever $\mathrm{H}$ is any non-transitive reflexive tournament. As $\mathrm{DC}_{3}^{*}$ is such a digraph, this proof also can be used for the case $\mathrm{H}=\mathrm{DC}_{3}^{*}$. However, it does not extend to Surjective $\mathrm{DC}_{k}^{*}$-Colouring for $k \geq 4$.

\section{A Dichotomy for Reflexive Tournaments}

In this section we prove our main result, namely a dichotomy of SuRJECTIVE H-COLOURING for reflexive tournaments $\mathrm{H}$ by showing that transitivity is the crucial property for tractability. In the next subsections we prove that SurJective H-Colouring is NP-complete when $\mathrm{H}$ is a non-transitive tournament.

\subsection{Two Elementary Lemmas}

It is well-known that every strongly connected tournament has a directed Hamilton cycle [6]. Hence we derive the following corollary to Lemmas 5 and 8 Part 2.

- Lemma 12. If $\mathrm{H}$ is a reflexive tournament that is endo-trivial, then $\mathrm{H}$ contains a directed Hamilton cycle.

We will also need the following lemma.

- Lemma 13. If $\mathrm{H}$ is a reflexive tournament that is endo-trivial, then any homomorphic image of $\mathrm{H}$ of size $1<n<|V(\mathrm{H})|$ possesses a double edge.

Proof. Suppose $\mathrm{H}$ has a homomorphic image of size $1<n<|V(\mathrm{H})|$ without a double edge. By looking at the equivalence classes of vertices identified in the homomorphic image, we can deduce a non-trivial retraction, namely by mapping each of the vertices in an equivalence class to any particular one of them.

\subsection{The NP-Hardness Gadget}

We now introduce the gadget $\mathrm{Cyl}_{m}^{*}$ drawn in Figure 3. We take $m$ disjoint copies of the directed $m$-cycle $\mathrm{DC}_{m}^{*}$ arranged in a cylindrical fashion so that there is an edge from $i$ in the $j$ th copy to $i$ in the $j+1$ th copy (drawn in red), and an edge from $i$ in the $j+1$ th copy to $i+1$ in the $j$ th copy (drawn in green). We consider $\operatorname{DC}_{m}^{*}$ to have vertices $\{1, \ldots, m\}$. A key role will be played by Hamilton cycles $\mathrm{HC}_{m}$ in a strongly connected reflexive tournament on $m$ vertices. We consider this cycle also labelled $\{1, \ldots, m\}$, in order to attach it to the gadget $\mathrm{Cyl}_{m}^{*}$. The gadget $\mathrm{Cyl}_{m}^{*}$ is an alteration of a gadget that appears in [9] for proving that LiST H-COLOURING is NP-complete when $\mathrm{H}$ is an undirected cycle on at least four vertices, but our proof is very different.

The following lemma follows from induction on the copies of $\mathrm{DC}_{m}^{*}$, since a reflexive tournament has no double edges. 


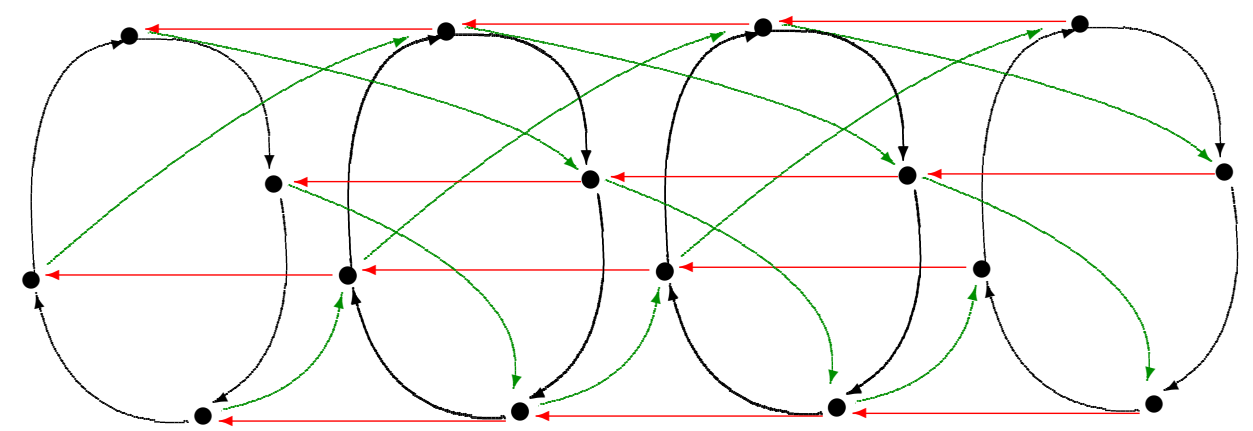

Figure 3 The gadget $\mathrm{Cyl}_{m}^{*}$ in the case $m:=4$ (self-loops are not drawn). We usually visualise the right-hand copy of $\mathrm{DC}_{4}^{*}$ as the "bottom" copy and then we talk about vertices "above" and "below" according to the red arrows.

- Lemma 14. In any homomorphism $h$ from $\mathrm{Cyl}_{m}^{*}$, with bottom cycle $\mathrm{DC}_{m}^{*}$, to a reflexive tournament, if $\left|h\left(\mathrm{DC}_{m}^{*}\right)\right|=1$, then $\left|h\left(\mathrm{Cyl}_{m}^{*}\right)\right|=1$.

We will use another property, denoted $(\dagger)$, of $\mathrm{Cyl}_{m}^{*}$, which is that the retractions from $\mathrm{Cyl}_{m}^{*}$ to its bottom copy of $\mathrm{DC}_{m}^{*}$, once propagated through the intermediate copies, induce on the top copy precisely the set of automorphisms of $\mathrm{DC}_{m}^{*}$. That is, the top copy of $\mathrm{DC}_{m}^{*}$ is mapped isomorphically to the bottom copy, and all such isomorphisms may be realised. The reason is that in such a retraction, the $(j+1)$ th copy may either map under the identity to the $j$ th copy, or rotate one edge of the cycle clockwise, and $\mathrm{Cyl}_{m}^{*}$ consists of sufficiently many (namely $m$ ) copies of $\mathrm{DC}_{m}^{*}$.

Now let $\mathrm{H}$ be a reflexive tournament that contains a subtournament $\mathrm{H}_{0}$ on $m$ vertices that is endo-trivial. By Lemma 12, we find that $\mathrm{H}_{0}$ contains at least one directed Hamilton cycle $\mathrm{HC}_{0}$. Define $\operatorname{Spill}_{m}\left(\mathrm{H}\left[\mathrm{H}_{0}, \mathrm{HC}_{0}\right]\right)$ as follows. Begin with $\mathrm{H}$ and add a copy of the gadget $\mathrm{Cyl} l_{m}^{*}$, where the bottom copy of $\mathrm{DC}_{m}^{*}$ is identified with $\mathrm{HC}_{0}$, to build a digraph $\mathrm{F}\left(\mathrm{H}_{0}, \mathrm{HC}_{0}\right)$. Now ask, for some $y \in V(\mathrm{H})$ whether there is a retraction $r$ of $\mathrm{F}\left(\mathrm{H}_{0}, \mathrm{HC}_{0}\right)$ to $\mathrm{H}$ so that some vertex $x$ in the top copy of $\mathrm{DC}_{m}^{*}$ in $\mathrm{Cyl}_{m}^{*}$ is such that $r(x)=y$. Such vertices $y$ comprise the set $\operatorname{Spill}_{m}\left(\mathrm{H}\left[\mathrm{H}_{0}, \mathrm{HC}_{0}\right]\right)$.

We now observe that $\operatorname{Spill}_{m}\left(\mathrm{H}\left[\mathrm{H}_{0}, \mathrm{HC}_{0}\right]\right)=V(\mathrm{H})$ if $\mathrm{H}$ retracts to $\mathrm{H}_{0}$.

- Lemma 15. If $\mathrm{H}$ is a reflexive tournament that retracts to a subtournament $\mathrm{H}_{0}$ with Hamilton cycle $\mathrm{HC}_{0}$, then $\operatorname{Spill}_{m}\left(\mathrm{H}\left[\mathrm{H}_{0}, \mathrm{HC}_{0}\right]\right)=V(\mathrm{H})$.

\subsection{Two Base Cases}

Recall that if $\mathrm{H}$ is an endo-trivial tournament, then SurJeCtive H-Colouring is NPcomplete due to Corollary 10. However $\mathrm{H}$ may not be endo-trivial. We will now show how to deal with the case where $\mathrm{H}$ is not endo-trivial but retracts to an endo-trivial subtournament. For doing this we use the above gadget, but we need to distinguish between two different cases.

- Lemma 16 (Base Case I.). Let $\mathrm{H}$ be a reflexive tournament that retracts to an endotrivial subtournament $\mathrm{H}_{0}$ with Hamilton cycle $\mathrm{HC}_{0}$. Assume that $\mathrm{H}$ retracts to $\mathrm{H}_{0}^{\prime}$ for every isomorphic copy $\mathrm{H}_{0}^{\prime}=i\left(\mathrm{H}_{0}\right)$ of $\mathrm{H}_{0}$ in $\mathrm{H}$ with $\operatorname{Spill}_{m}\left(\mathrm{H}\left[\mathrm{H}_{0}^{\prime}, i\left(\mathrm{HC}_{0}\right)\right]\right)=V(\mathrm{H})$. Then $\mathrm{H}_{0}$-REtraction can be polynomially reduced to SurJeCtive H-COLOURING.

Proof. Let $\mathrm{G}$ be an instance of $\mathrm{H}_{0}$-Retraction. We build an instance $\mathrm{G}^{\prime \prime}$ of SuRJective H-Colouring in the following fashion. First, take a copy of $\mathrm{H}$ together with $\mathrm{G}$ and build 


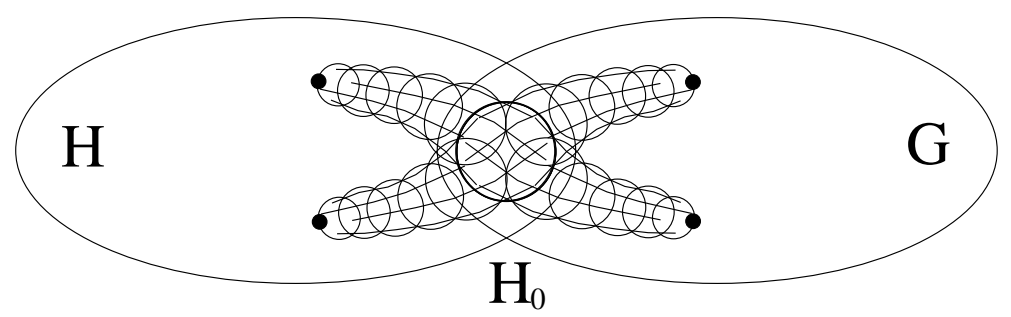

Figure 4 A stylised depiction of the construction in Base Case I. The central circle is the Hamilton cycle and the eccentric circles emanating thereout are the gadgets $\mathrm{Cyl}_{m}^{*}$.

$\mathrm{G}^{\prime}$ by identifying these on the copy of $\mathrm{H}_{0}$ that they both possess as a subgraph. Let $m$ be the size of $\mathrm{H}_{0}$ and consider its Hamilton cycle $\mathrm{HC}_{0}$. We build $\mathrm{G}^{\prime \prime}$ from $\mathrm{G}^{\prime}$ by augmenting a new copy of $\mathrm{Cyl}_{m}^{*}$ for every vertex $v \in V\left(\mathrm{G}^{\prime}\right) \backslash V\left(\mathrm{H}_{0}\right)$. Vertex $v$ is to be identified with any vertex in the top copy of $\mathrm{DC}_{m}^{*}$ in $\mathrm{Cyl}_{m}^{*}$ and the bottom copy of $\mathrm{DC}_{m}^{*}$ is to be identified with $\mathrm{HC}_{0}$ in $\mathrm{H}_{0}$ according to the identity function. See Figure 4 for an example. We claim that $\mathrm{G}$ retracts to $\mathrm{H}_{0}$ if and only if there exists a surjective homomorphism from $\mathrm{G}^{\prime \prime}$ to $\mathrm{H}$.

First suppose that $\mathrm{G}$ retracts to $\mathrm{H}_{0}$. Let $h$ be a retraction from $\mathrm{G}$ to $\mathrm{H}_{0}$. We extend $h$ as follows. First we map the copy of $\mathrm{H}$ in $\mathrm{G}^{\prime \prime}$ to itself in $\mathrm{H}$ by the identity. This will ensure surjectivity. We then map the various copies of $\mathrm{Cyl}_{m}^{*}$ in $\mathrm{G}^{\prime \prime}$. This is always possible: because $\mathrm{H}$ retracts to $\mathrm{H}_{0}$, we have $\operatorname{Spill}_{m}\left(\mathrm{H}_{[}\left[\mathrm{H}_{0}, \mathrm{HC}_{0}\right]\right)=V(\mathrm{H})$ due to Lemma 15. Hence, if $h(x)=y$ for two vertices $x \in V\left(\mathrm{G}^{\prime}\right) \backslash V\left(\mathrm{H}_{0}\right)$ and $y \in V(\mathrm{H})$, we can always find a retraction of the graph $\mathrm{F}\left(\mathrm{H}_{0}, \mathrm{HC}_{0}\right)$ to $\mathrm{H}$ that maps $x$ to $y$, and we mimic this retraction on the corresponding subgraph in $\mathrm{G}^{\prime \prime}$. The crucial observation is that this can be done independently for each vertex in $V\left(\mathrm{G}^{\prime}\right) \backslash V\left(\mathrm{H}_{0}\right)$, as two vertices of different copies of $\mathrm{Cyl}_{m}^{*}$ are only adjacent if they both belong to $\mathrm{G}^{\prime}$. This leads to a surjective homomorphism from $\mathrm{G}^{\prime \prime}$ to $\mathrm{H}$.

Now suppose that there exists a surjective homomorphism $h$ from $\mathrm{G}^{\prime \prime}$ to $\mathrm{H}$. If $\left|h\left(\mathrm{H}_{0}\right)\right|=1$, then by Lemma 14, $\left|h\left(\mathrm{Cyl}_{m}^{*}\right)\right|=1$ for all copies of $\mathrm{Cyl}_{m}^{*}$ in $\mathrm{G}^{\prime \prime}$. This means that $\left|h\left(\mathrm{G}^{\prime \prime}\right)\right|=1$ and $h$ is not surjective, a contradiction. Now, $1<\left|h\left(\mathrm{H}_{0}\right)\right|<m$ is not possible either due to Lemma 13. Thus, $\left|h\left(\mathrm{H}_{0}\right)\right|=m$ and indeed $h$ maps $\mathrm{H}_{0}$ to a copy of itself in $\mathrm{H}$ which we will call $\mathrm{H}_{0}^{\prime}=i\left(\mathrm{H}_{0}\right)$ for some isomorphism $i$.

We claim that $\operatorname{Spill}_{m}\left(\mathrm{H}\left[\mathrm{H}_{0}^{\prime}, i\left(\mathrm{HC}_{0}\right)\right]\right)=V(\mathrm{H})$. In order to see this, consider a vertex $y \in V(\mathrm{H})$. As $h$ is surjective, there exists a vertex $x \in V\left(\mathrm{G}^{\prime \prime}\right)$ with $h(x)=y$. By construction, $x$ belongs to some copy of $\mathrm{DC}_{m}^{*}$, and thus also belongs to some copy of $\mathrm{DC}_{m}^{*}$ in $\mathrm{F}\left(\mathrm{H}_{0}, \mathrm{HC}_{0}\right)$. We can extend $i^{-1}$ to an isomorphism from the copy of $\mathrm{Cyl}_{m}^{*}$ (which has $i\left(\mathrm{HC}_{0}\right)$ as its bottom cycle) in the graph $\mathrm{F}\left(\mathrm{H}_{0}^{\prime}, i\left(\mathrm{HC}_{0}\right)\right)$ to the copy of $\mathrm{Cyl}_{m}^{*}$ (which has $\mathrm{HC}_{0}$ as its bottom cycle) in the graph $\mathrm{F}\left(\mathrm{H}_{0}, \mathrm{HC}_{0}\right)$. We define a mapping $r^{*}$ from $\mathrm{F}\left(\mathrm{H}_{0}^{\prime}, i\left(\mathrm{HC}_{0}\right)\right)$ to $\mathrm{H}$ by $r^{*}(u)=h \circ i^{-1}(u)$ if $u$ is on the copy of $\mathrm{Cyl}_{m}^{*}$ in $\mathrm{F}\left(\mathrm{H}_{0}^{\prime}, i\left(\mathrm{HC}_{0}\right)\right)$ and $r^{*}(u)=u$ otherwise. We observe that $r^{*}(u)=u$ if $u \in V\left(\mathrm{H}_{0}^{\prime}\right)$ as $h$ coincides with $i$ on $\mathrm{H}_{0}$. As $\mathrm{H}_{0}$ separates the other vertices of the copy of $\mathrm{Cyl}_{m}^{*}$ from $V(\mathrm{H}) \backslash V\left(\mathrm{H}_{0}\right)$, in the sense that removing $\mathrm{H}_{0}$ would disconnect them, this means that $r^{*}$ is a retraction from $\mathrm{F}\left(\mathrm{H}_{0}^{\prime}, i\left(\mathrm{HC}_{0}\right)\right)$ to $\mathrm{H}$. We find that $r^{*}$ maps $i(x)$ to $h \circ i^{-1}(i(x))=h(x)=y$. Moreover, as $x$ is in some copy of $\mathrm{DC}_{m}^{*}$ in $\mathrm{F}\left(\mathrm{H}_{0}, \mathrm{HC}_{0}\right)$, we have that $i(x)$ is in some copy of $\mathrm{DC}_{m}^{*}$ in $\mathrm{F}\left(\mathrm{H}_{0}^{\prime}, i\left(\mathrm{HC}_{0}\right)\right)$. We may assume without loss of generality that $i(x)$ belongs to the top copy. We conclude that $y$ always belongs to $\operatorname{Spill}_{m}\left(\mathrm{H}\left[\mathrm{H}_{0}^{\prime}, i\left(\mathrm{HC}_{0}\right)\right]\right)$.

As Spill $m\left(\mathrm{H}\left[\mathrm{H}_{0}^{\prime}, i\left(\mathrm{HC}_{0}\right)\right]\right)=V(\mathrm{H})$, we find, by assumption of the lemma, that there exists a retraction $r$ from $\mathrm{H}$ to $\mathrm{H}_{0}^{\prime}$. Now $i^{-1} \circ r \circ h$ ia the desired retraction of $\mathrm{G}$ to $\mathrm{H}_{0}$.

We now need to deal with the situation in which we have an isomorphic copy $\mathrm{H}_{0}^{\prime}=i\left(\mathrm{H}_{0}\right)$ of $\mathrm{H}_{0}$ in $\mathrm{H}$ with $\operatorname{Spill}_{m}\left(\mathrm{H}\left[\mathrm{H}_{0}^{\prime}, i\left(\mathrm{HC}_{0}\right)\right]\right)=V(\mathrm{H})$, such that $\mathrm{H}$ does not retract to $\mathrm{H}_{0}^{\prime}$ (see 


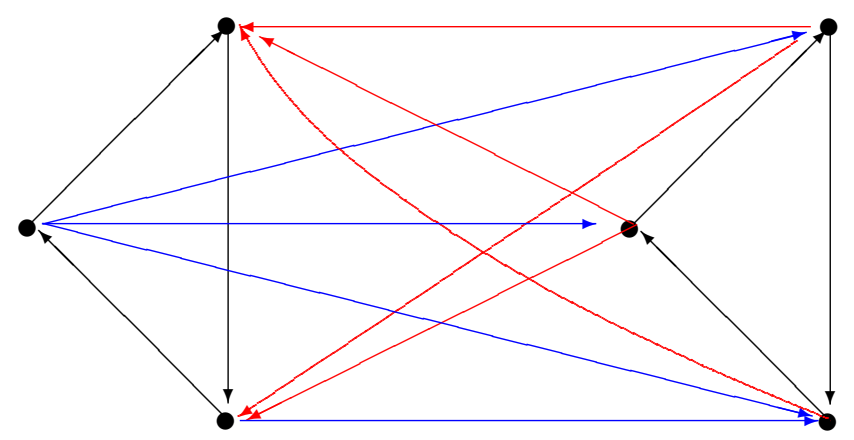

Figure $5 \mathrm{An}$ interesting tournament $\mathrm{H}$ on six vertices (self-loops are not drawn). This tournament does not retract to the $\mathrm{DC}_{3}^{*}$ on the left-hand side, yet $\operatorname{Spill}_{3}\left(\mathrm{H}\left[\mathrm{DC}_{3}^{*}, \mathrm{DC}_{3}\right]\right)=V(\mathrm{H})$.

Figure 5 for an example). We cannot deal with this case in a direct matter and first show another base case. For this we need the following lemma and an extension of endo-triviality that we discuss afterwards.

- Lemma 17. Let $\mathrm{H}$ be a reflexive tournament, containing a subtournament $\mathrm{H}_{0}$ so that any endomorphism of $\mathrm{H}$ that fixes $\mathrm{H}_{0}$ is an automorphism. Then any endomorphism of $\mathrm{H}$ that maps $\mathrm{H}_{0}$ to an isomorphic copy $\mathrm{H}_{0}^{\prime}=i\left(\mathrm{H}_{0}\right)$ of itself is an automorphism of $\mathrm{H}$.

Proof. For contradiction, suppose there is an endomorphism $h$ that maps $\mathrm{H}_{0}$ to an isomorphic copy $\mathrm{H}_{0}^{\prime}=i\left(\mathrm{H}_{0}\right)$ of itself that is not an automorphism of $\mathrm{H}$. In particular, $|h(\mathrm{H})|<|V(\mathrm{H})|$. Choose $h^{-1}$ in the following fashion. We let $h^{-1}$ of $h\left(\mathrm{H}_{0}\right)$ be the natural isomorphism of $h\left(\mathrm{H}_{0}\right)$ to $\mathrm{H}_{0}$ (that inverts the isomorphism given by $h$ from $\mathrm{H}_{0}$ to $\mathrm{H}_{0}^{\prime}$ ). Otherwise we choose $h^{-1}$ arbitrarily, such that $h^{-1}(y)=x$ only if $h(x)=y$. Since $\mathrm{H}$ is a reflexive tournament, containing precisely one edge between distinct vertices, $h^{-1}$ is an isomorphism. Moreover, $h^{-1} \circ h$ is an endomorphism of $\mathrm{H}$ that fixes $\mathrm{H}_{0}$ and that is not an automorphism, a contradiction.

Let $\mathrm{H}_{0}$ be an induced subgraph of a digraph $\mathrm{H}$. We say that the pair $\left(\mathrm{H}, \mathrm{H}_{0}\right)$ is endo-trivial if all endomorphisms of $\mathrm{H}$ that fix $\mathrm{H}_{0}$ are automorphisms.

- Lemma 18 (Base Case II). Let $\mathrm{H}$ be a reflexive tournament with a subtournament $\mathrm{H}_{0}$ with Hamilton cycle $\mathrm{HC}_{0}$ so that $\left(\mathrm{H}, \mathrm{H}_{0}\right)$ and $\mathrm{H}_{0}$ are endo-trivial and $\operatorname{Spill}_{m}\left(\mathrm{H}\left[\mathrm{H}_{0}, \mathrm{HC}_{0}\right]\right)=V(\mathrm{H})$. Then H-Retraction can be polynomially reduced to SurJective H-Colouring.

\subsection{Generalising the Base Cases}

We now generalise the two base cases to more general cases via some recursive procedure. Afterwards we will show how to combine these two cases to complete our proof. We will first need a slightly generalised version of Lemma 17, which nonetheless has virtually the same proof.

- Lemma 19. Let $\mathrm{H}_{2} \supset \mathrm{H}_{1} \supset H_{0}$ be a sequence of strongly connected reflexive tournaments, each one a subtournament of the one before. Suppose that any endomorphism of $\mathrm{H}_{1}$ that fixes $\mathrm{H}_{0}$ is an automorphism. Then any endomorphism $h$ of $\mathrm{H}_{2}$ that maps $\mathrm{H}_{0}$ to an isomorphic copy $\mathrm{H}_{0}^{\prime}=i\left(\mathrm{H}_{0}\right)$ of itself also gives an isomorphic copy of $\mathrm{H}_{1}$ in $h\left(\mathrm{H}_{1}\right)$.

The following two lemmas generalize Lemmas 16 and 18. 
- Lemma 20 (General Case I). Let $\mathrm{H}_{0}, \mathrm{H}_{1}, \ldots, \mathrm{H}_{k}, \mathrm{H}_{k+1}$ be reflexive tournaments, the first $k$ of which have Hamilton cycles $\mathrm{HC}_{0}, \mathrm{HC}_{1}, \ldots, \mathrm{HC}_{k}$, respectively, so that $\mathrm{H}_{0} \subseteq H_{1} \subseteq \cdots \subseteq$ $\mathrm{H}_{k} \subseteq \mathrm{H}_{k+1}$. Assume that $\mathrm{H}_{0},\left(\mathrm{H}_{1}, \mathrm{H}_{0}\right), \ldots,\left(\mathrm{H}_{k}, \mathrm{H}_{k-1}\right)$ are endo-trivial and that

$$
\begin{array}{lcc}
\text { Spill }_{a_{0}}\left(\mathrm{H}_{1}\left[\mathrm{H}_{0}, \mathrm{HC}_{0}\right]\right) & = & V\left(\mathrm{H}_{1}\right) \\
\operatorname{Spill}_{a_{1}}\left(\mathrm{H}_{2}\left[\mathrm{H}_{1}, \mathrm{HC}_{1}\right]\right) & = & V\left(\mathrm{H}_{2}\right) \\
\vdots & \vdots & \vdots \\
\text { Spill }_{a_{k-1}}\left(\mathrm{H}_{k}\left[\mathrm{H}_{k-1}, \mathrm{HC}_{k-1}\right]\right) & = & V\left(\mathrm{H}_{k}\right) .
\end{array}
$$

Assume that $\mathrm{H}_{k+1}$ retracts to $\mathrm{H}_{k}$ and also to every isomorphic copy $\mathrm{H}_{k}^{\prime}=i\left(\mathrm{H}_{k}\right)$ of $\mathrm{H}_{k}$ in $\mathrm{H}_{k+1}$ with Spill $a_{k}\left(\mathrm{H}_{k+1}\left[\mathrm{H}_{k}^{\prime}, i\left(\mathrm{HC}_{k}\right)\right]\right)=V\left(\mathrm{H}_{k+1}\right)$. Then $\mathrm{H}_{k}$-RETRACTION can be polynomially reduced to SurJective $\mathrm{H}_{k+1}$-COLOURING.

- Lemma 21 (General Case II). Let $\mathrm{H}_{0}, \mathrm{H}_{1}, \ldots, \mathrm{H}_{k}, \mathrm{H}_{k+1}$ be reflexive tournaments, the first $k+1$ of which have Hamilton cycles $\mathrm{HC}_{0}, \mathrm{HC}_{1}, \ldots, \mathrm{HC}_{k}$, respectively, so that $\mathrm{H}_{0} \subseteq H_{1} \subseteq$ $\cdots \subseteq \mathrm{H}_{k} \subseteq \mathrm{H}_{k+1}$. Suppose that $\mathrm{H}_{0},\left(\mathrm{H}_{1}, \mathrm{H}_{0}\right), \ldots,\left(\mathrm{H}_{k}, \mathrm{H}_{k-1}\right),\left(\mathrm{H}_{k+1}, \mathrm{H}_{k}\right)$ are endo-trivial and that

$$
\begin{array}{lcc}
\operatorname{Spill}_{a_{0}}\left(\mathrm{H}_{1}\left[\mathrm{H}_{0}, \mathrm{HC}_{0}\right]\right) & = & V\left(\mathrm{H}_{1}\right) \\
\operatorname{Spill}_{a_{1}}\left(\mathrm{H}_{2}\left[\mathrm{H}_{1}, \mathrm{HC}_{1}\right]\right) & = & V\left(\mathrm{H}_{2}\right) \\
\vdots & \vdots & \vdots \\
\text { Spill }_{a_{k-1}}\left(\mathrm{H}_{k}\left[\mathrm{H}_{k-1}, \mathrm{HC}_{k-1}\right]\right) & = & V\left(\mathrm{H}_{k}\right) \\
\text { Spill }_{a_{k}}\left(\mathrm{H}_{k+1}\left[\mathrm{H}_{k}, \mathrm{HC}_{k}\right]\right) & = & V\left(\mathrm{H}_{k+1}\right)
\end{array}
$$

Then $\mathrm{H}_{k+1}$-Retraction can be polynomially reduced to SurJeCtive $\mathrm{H}_{k+1}$-Colouring.

\subsection{Final Steps for Hardness for Non-Transitive Reflexive Tournaments}

We first prove, by using the lemmas from Section 4.4, that SurJective H-Colouring is $\mathrm{NP}$-complete if $\mathrm{H}$ is a non-transitive reflexive tournament that is strongly connected. For our discourse it is not necessary to know precisely what is a Taylor operation, but we will use the following result.

- Theorem 22 ([5, 22]). Let $\mathrm{H}$ be a finite structure so that the idempotent polymorphisms of $\mathrm{H}$ omit all Taylor operations. Then H-RETRACTION is NP-complete.

- Corollary 23. Let $\mathrm{H}$ be a strongly connected reflexive tournament. Then SURJECTIVE H-Colouring is NP-complete.

In order to deal with reflexive tournaments that are not strongly connected we need the following strengthened version of Corollary 23.

- Corollary 24. Let $\mathrm{H}$ be a strongly connected reflexive tournament. Then SURJECTIVE $\mathrm{H}$-COLOURING is NP-complete even for strongly connected digraphs.

We now give our main hardness result following with our main dichotomy.

- Theorem 25. Let $\mathrm{H}$ be a non-transitive reflexive tournament. Then SuRJECTIVE HColouring is NP-complete.

- Corollary 26. Let $\mathrm{H}$ be a reflexive tournament. If $\mathrm{H}$ is transitive, then SURJECTIVE $\mathrm{H}$-ColOuRING is in NL; otherwise it is NP-complete.

Proof. For the transitive case we can say that H-Retraction is in NL from [8], since $\mathrm{H}$ enjoys the ternary median operation as a polymorphism (this has been observed, inter alia, in [19]). It follows of course that SurJective H-Colouring is in NL also. The non-transitive case follows from Theorem 25. 


\section{Digraphs with a most three vertices}

- Theorem 27. Let $\mathrm{H}$ be a partially reflexive digraph of size at most 3. Then SURJECTIVE $\mathrm{H}$-COLOURING is polynomially equivalent to $\mathrm{H}$-RETRACTION. In particular, it is always in $\mathrm{P}$ or is NP-complete.

\section{Conclusion}

We have given the first significant classification results for SurJective H-Colouring where $\mathrm{H}$ comes from a class of digraphs (that are not graphs). To do this, we have developed both a novel algebraic method and a novel recursive combinatorial method. Below we discuss some directions for future research.

Let $3 \mathrm{NRC}$ be the hypergraph with vertex-set $\{r, g, b\}$ and hyperedge-set $\{r, g, b\} \backslash$ $\{(r, g, b),(r, b, g),(g, b, r),(g, r, b),(b, r, g),(b, g, r)\}$. Then 3-No-RainBow-Colouring is the problem SURJECTIVE 3NRC-COLOURING, in which one looks for a surjective colouring of the vertices, such that no hyperedge is rainbow-coloured (i.e. uses all colours). We recall that the complexity of this problem is open since it arose (under a different name) in [18], see also Question 3 in [2]. The SurJective DC $_{3}^{*}$-Colouring problem is the digraph problem most closely related to 3-No-RAINBOW-CoLOURING. To explain this, when looking for digraphs with a similar character to $3 \mathrm{NRC}$, we would insist at least that the automorphism group is transitive. This leaves just the reflexive and irreflexive directed 3-cycles and the reflexive and irreflexive 3 -cliques, that is, 3 -cycles with a double edge between every pair of vertices (admittedly, the cycles have only some of the automorphisms of the cliques). If $\mathrm{H}$ is the reflexive 3-clique, then H-Retraction and Surjective H-Colouring are trivial. If $\mathrm{H}$ is the irreflexive directed 3-cycle, then $\mathrm{H}$ has a majority polymorphism, which shows that H-Retraction, and thus Surjective H-Colouring (see Figure 1), can be solved in polynomial time [1]. If $\mathrm{H}$ is the irreflexive 3 -clique, then SurJeCtive H-Colouring is NPcomplete, as there exists a straightforward reduction from 3-ColOuRING. Hence $\mathrm{H}=\mathrm{DC}_{3}^{*}$ was indeed the only case for which determining the complexity of SURJECTIVE H-Colouring was not immediately obvious.

It would be great to extend our results to larger reflexive digraph classes. Reflexive digraphs with a double edge are not endo-trivial and further fail to be endo-trivial in the worse way, since SURJECTIVE $\mathrm{DC}_{2}^{*}$-COLOURING is nearly trivial. Thus, our methods are likely only to be applicable to reflexive oriented digraphs, that is, those without a double edge. On the way, a natural question arising is exactly which reflexive digraphs are endo-trivial?

Finally, there is the question as to whether the assumption of endo-triviality can be weakened to that of retract-triviality in Theorem 9. Endo-triviality is used right at the beginning of the proof to show that $\mathrm{G}^{\mathrm{G}}$ is the disjoint union of a copy of $\mathrm{G}$ (the constant maps) and isolated automorphisms. We do not know if retract-triviality is here sufficient.

\section{References}

1 Jørgen Bang-Jensen, Pavol Hell, and Gary MacGillivray. The complexity of colouring by semicomplete digraphs. SIAM Journal on Discrete Mathematics, 1(3):281-298, 1988.

2 Manuel Bodirsky, Jan Kára, and Barnaby Martin. The complexity of surjective homomorphism problems - a survey. Discrete Applied Mathematics, 160(12):1680-1690, 2012.

3 Andrei A. Bulatov. Complexity of conservative constraint satisfaction problems. ACM Transactions on Computational Logic, 12(4):24:1-24:66, 2011. 
4 Andrei A. Bulatov. A dichotomy theorem for nonuniform CSPs. Proc. FOCS 2017, pages 319-330, 2017.

5 Andrei A. Bulatov, Peter Jeavons, and Andrei A. Krokhin. Classifying the complexity of constraints using finite algebras. SIAM Journal on Computing, 34(3):720-742, 2005.

6 Paul Camion. Chemins et circuits hamiltoniens de graphes complets. C. R. Acad. Sci. Paris, 249:2151-2152, 1959.

7 Hubie Chen. An algebraic hardness criterion for surjective constraint satisfaction. Algebra Universalis, 72(4):393-401, 2014.

8 Víctor Dalmau and Andrei A. Krokhin. Majority constraints have bounded pathwidth duality. European Journal of Combinatorics, 29(4):821-837, 2008.

9 Tomás Feder and Pavol Hell. List homomorphisms to reflexive graphs. Journal of Combinatorial Theory, Series B, 72(2):236-250, 1998.

10 Tomás Feder, Pavol Hell, and Jing Huang. List homomorphisms and circular arc graphs. Combinatorica, 19(4):487-505, 1999.

11 Tomás Feder, Pavol Hell, and Jing Huang. Bi-arc graphs and the complexity of list homomorphisms. Journal of Graph Theory, 42(1):61-80, 2003.

12 Tomás Feder, Pavol Hell, Peter Jonsson, Andrei A. Krokhin, and Gustav Nordh. Retractions to pseudoforests. SIAM Journal on Discrete Mathematics, 24(1):101-112, 2010.

13 Tomás Feder, Pavol Hell, and Kim Tucker-Nally. Digraph matrix partitions and trigraph homomorphisms. Discrete Applied Mathematics, 154(17):2458-2469, 2006.

14 Tomás Feder and Moshe Y. Vardi. The computational structure of monotone monadic SNP and constraint satisfaction: A study through datalog and group theory. SIAM Journal on Computing, 28(1):57-104, 1998.

15 Petr A. Golovach, Matthew Johnson, Barnaby Martin, Daniël Paulusma, and Anthony Stewart. Surjective $H$-colouring: New hardness results. Proc. CiE 2017, Lecture Notes in Computer Science, 10307:270-281, 2017.

16 Petr A. Golovach, Daniël Paulusma, and Jian Song. Computing vertex-surjective homomorphisms to partially reflexive trees. Theoretical Computer Science, 457:86-100, 2012.

17 David Charles Hobby and Ralph McKenzie. The Structure of Finite Algebras, Contemporary Mathematics. American Mathematical Society, 1988.

18 Daniel Král, Jan Kratochvíl, Andrzej Proskurowski, and Heinz-Jürgen Voss. Coloring mixed hypertrees. Discrete Applied Mathematics, 154(4):660-672, 2006.

19 Benoit Larose. Taylor operations on finite reflexive structures. International Journal of Mathematics and Computer Science, 1(1):1-26, 2006.

20 Benoit Larose. Algebra and the complexity of digraph CSPs: a survey. In Andrei A. Krokhin and Stanislav Zivny, editors, The Constraint Satisfaction Problem: Complexity and Approximability, volume 7 of Dagstuhl Follow-Ups, pages 267-285. Schloss Dagstuhl Leibniz-Zentrum fuer Informatik, 2017.

21 Benoit Larose, Cynthia Loten, and Claude Tardif. A characterisation of first-order constraint satisfaction problems. Logical Methods in Computer Science, 3(4), 2007.

22 Benoit Larose and László Zádori. Finite posets and topological spaces in locally finite varieties. Algebra Universalis, 52(2):119-136, 2005.

23 Miklós Maróti and László Zádori. Reflexive digraphs with near unanimity polymorphisms. Discrete Mathematics, 312(15):2316-2328, 2012.

24 Barnaby Martin and Daniël Paulusma. The computational complexity of disconnected cut and $2 \mathrm{k} 2$-partition. Journal of Combinatorial Theory, Series B, 111:17-37, 2015.

25 Mark Siggers. personal communication.

26 Agnes Szendrei. Term minimal algebras. Algebra Universalis, 32(4):439-477, 1994.

27 Narayan Vikas. Computational complexity of compaction to reflexive cycles. SIAM Journal on Computing, 32(1):253-280, 2002. 
28 Narayan Vikas. Computational complexity of compaction to irreflexive cycles. Journal of Computer and System Sciences, 68(3):473-496, 2004.

29 Narayan Vikas. A complete and equal computational complexity classification of compaction and retraction to all graphs with at most four vertices and some general results. Journal of Computer and System Sciences, 71(4):406-439, 2005.

30 Narayan Vikas. Algorithms for partition of some class of graphs under compaction and vertex-compaction. Algorithmica, 67(2):180-206, 2013.

31 Narayan Vikas. Computational complexity of graph partition under vertex-compaction to an irreflexive hexagon. In Kim G. Larsen, Hans L. Bodlaender, and Jean-François Raskin, editors, 42nd International Symposium on Mathematical Foundations of Computer Science, MFCS 2017, August 21-25, 2017 - Aalborg, Denmark, volume 83 of LIPIcs, pages 69:1-69:14. Schloss Dagstuhl - Leibniz-Zentrum fuer Informatik, 2017.

32 Dmitriy Zhuk. A proof of CSP dichotomy conjecture. Proc. FOCS 2017, pages 331-342, 2017. 